\title{
Injeção intravítrea de acetato de triancinolona no tratamento da síndrome de Vog't-Koyanagi-Harada
}

\author{
Intravitreal triamcinolone acetonide injection in the treatment of \\ Vogt-Koyanagi-Harada syndrome
}

\author{
Rafael Ernane Almeida Andrade ${ }^{1}$ \\ Cristina Muccioli² \\ Michel Eid Farah ${ }^{3}$
}

Trabalho realizado Instituto da Visão, Departamento de Oftalmologia - Universidade Federal de São Paulo.

${ }^{1}$ Pós-graduando, nível doutorado pela Universidade Federal de São Paulo - UNIFESP.

${ }^{2}$ Professora afiliada, Livre Docente e chefe do Setor de Uveítes e AIDS da Universidade Federal de São Paulo UNIFESP.

${ }^{3}$ Professor Livre Docente do Departamento de Oftalmologia da Universidade Federal de São Paulo - UNIFESP.

Endereço para correspondência: Rafael Ernane Almeida Andrade, Av: Onze de Junho, 685, Ap 43, B1 1,

São Paulo (SP) - CEP 04041-052

E-mail: rafaelernane@uol.com.br

Recebido para publicação em 17.12.2003

Versão revisada recebida em 02.03.2004

Aprovação em 18.03.2004

\begin{tabular}{|l|}
\hline RESUMO \\
\hline Objetivo: Avaliar o uso da injeção intravítrea do acetato de triancinolona \\
no tratamento da fase aguda da síndrome de Vogt-Koyanagi-Harada, \\
demonstrando a rápida resolução do descolamento seroso de retina. \\
Métodos: Nove olhos de cinco pacientes apresentando descolamento \\
seroso de retina associado à síndrome de Vogt-Koyanagi-Harada foram \\
tratados com uma única injeção intravítrea de 4 mg de acetato triancinolona. \\
Os seguintes parâmetros foram avaliados: melhor acuidade visual, pressão \\
intra-ocular e a altura do descolamento de acordo com a tomografia de \\
coerência óptica. Resultados: Em todos os olhos a tomografia de coerência \\
óptica revelou diminuição marcada no descolamento seroso de retina na \\
primeira semana após a injeção intravítrea do acetato de triancinolona, com \\
subseqüente recuperação da acuidade visual e das características anatô- \\
micas retinianas normais. Não foram observadas complicações durante o \\
seguimento, que variou de 5 a 12 meses (média de 7,8 meses). Conclusões: \\
A injeção intravítrea do acetato de triancinolona pode proporcionar em \\
curto tempo a resolução do processo inflamatório e exsudativo intra e sub- \\
retiniano na síndrome de Vogt-Koyanagi-Harada cursando com melhora \\
da acuidade visual. São necessários novos estudos para avaliar a eficácia \\
e a segurança deste tipo de procedimento a longo prazo.
\end{tabular}

Descritores: Descolamento retiniano; Síndrome uveomeningoencefálica/quimioterapia; Triancinolona/uso terapêutico; Tomografia de coerência óptica

\section{INTRODUÇÃ̃O}

A síndrome de Vogt-Koynagi-Harada é uma doença inflamatória, também chamada de síndrome uveomeníngea, caracterizando-se por apresentar uveíte difusa bilateral, geralmente granulomatosa, de etiologia desconhecida, acompanhada por descolamento exsudativo de retina, além de manifestações dermatológicas, meníngeas e auditivas ${ }^{(1-3)}$. No Brasil, representa 2,5\% do total de uveítes, enquanto nos Estados Unidos esta incidência é de aproximadamente $0,9 \%$ e no Japão de $8,0 \%{ }^{(4)}$.

O prognóstico visual dos pacientes é reservado, relacionado principalmente ao desenvolvimento de glaucoma e membrana neovascular subretiniana na fase crônica. A acuidade visual final melhor do que 20/40 ocorre em apenas $30 \%$ dos casos, tratando-se, portanto, de importante causa de cegueira $^{(5-6)}$.

Geralmente a doença inicia com descolamentos serosos da retina, bilaterais, associados à inflamação vítrea e da câmara anterior podendo persistir por vários meses após o início dos sintomas ${ }^{(1)}$. Altas doses de corticotera- 
pia sistêmica, particularmente a pulsoterapia com o uso de medicação endovenosa, têm sido a terapia de escolha para a síndrome de Vogt-Koyanagi-Harada. Este tipo de tratamento associado ao longo período de uso, inevitavelmente, está relacionado a uma série de efeitos colaterais sistêmicos, muitas vezes graves, como a supressão do sistema imune e a síndrome de Cushing ${ }^{(1-3,6)}$.

Com o objetivo de diminuir os efeitos sistêmicos e o tempo de sofrimento ocular, a triancinolona intravítrea vem se consolidando como uma boa alternativa de tratamento para as complicações associadas às uveítes, principalmente no tratamento do edema cistóide de mácula onde se acumula a maior experiência ${ }^{(7-9)}$. Este trabalho tem o objetivo de avaliar o uso do acetato de triancinolona intravítreo em pacientes com descolamento seroso de retina associado à síndrome de Vogt-Koyanagi-Harada.

\section{MÉTODOS}

Um total de nove olhos de cinco pacientes com o diagnóstico da síndrome de Vogt-Koyanagi-Harada e que apresentavam descolamento seroso de retina foram incluídos neste estudo não comparativo, prospectivo e intervencional. Esses pacientes foram examinados no Departamento de Oftalmologia da Universidade Federal de São Paulo (UNIFESP). O estudo foi aprovado pelo Comitê de Ética da UNIFESP e todos os pacientes assinaram previamente o termo de consentimento livre esclarecido.

Foi utilizada a dose de $4 \mathrm{mg} / 0,1 \mathrm{ml}$ de acetato de triancinolona intravítreo. A medicação sistêmica (Prednisona $40 \mathrm{mg}$ ) foi prescrita somente nos pacientes com sintomas sistêmicos, sendo feita a diminuição progressiva da dose logo após a injeção intravítrea. Os parâmetros de sucesso medidos foram a acuidade visual, a pressão intra-ocular e a avaliação quantitativa do descolamento da retina na região macular por meio da tomografia de coerência óptica ${ }^{(9)}$.

A tomografia de coerência óptica de alta resolução (Stratus OCT), exame não invasivo, que realiza cortes seccionais da retina e coróide foi realizada em todos os pacientes imediatamente antes da injeção, uma semana e um mês após o trata- mento. As imagens obtidas foram centradas na depressão foveal. Utilizando o programa de medida da espessura da retina (retinal thickness), foi medida a altura do descolamento da retina no centro da fóvea.

\section{RESULTADOS}

Dos cinco pacientes incluídos no estudo, quatro eram mulheres (80\%), e a idade variou de 35 a 56 anos (média de 49 anos). Três dos pacientes $(60 \%)$ apresentavam hipertensão arterial sistêmica, controlada com medicação, sendo que uma paciente também era diabética. Somente o paciente 1 apresentou sinais sistêmicos de meningismo associados ao quadro ocular (Tabela 1).

Todos os pacientes foram tratados bilateralmente com uma única injeção intravítrea de $4 \mathrm{mg}$ de acetato de triancinolona, com exceção do paciente 1, onde somente o olho direito, com sintomas mais graves, foi submetido à injeção, demonstrando uma rápida melhora com a resolução total do descolamento na primeira semana, enquanto que no olho contralateral, que foi tratado somente com a medicação sistêmica, o descolamento de retina e a papilite duraram em torno de dois meses.

Os pacientes 1 , 3, e 5 estavam em uso de $1 \mathrm{mg} / \mathrm{kg} / \mathrm{dia}$ de prednisona oral, sendo diminuída progressivamente a dose logo após a injeção intravítrea do acetato de triancinolona, até a sua retirada total em torno de 3 meses (Figura 1). Os casos 2 e 4 foram tratados somente com a injeção intravítrea de triancinolona, apresentando melhora total do descolamento de retina e da papilite em aproximadamente uma semana. Porém a paciente 2 apresentou disacusia no segundo mês de seguimento, após a resolução do quadro ocular, sendo introduzido $40 \mathrm{mg}$ de prednisona oral, evoluindo com a melhora completa dos sintomas com 1 semana, sendo então iniciado a diminuição progressiva da medicação sistêmica após 3 semanas. A paciente 4 se encontra no sexto mês de seguimento sem recidiva do quadro inflamatório (Figuras 2 e 3 ).

O paciente 5 apresentava um quadro raro de descolamento seroso de retina recidivado pela terceira vez, atingindo mais intensamente o olho direito, associado a um edema cistóide

\begin{tabular}{|c|c|c|c|c|c|}
\hline & Paciente 1 & Paciente 2 & Paciente 3 & Paciente 4 & Paciente 5\# \\
\hline Idade (anos) & 56 & 48 & 56 & 35 & 50 \\
\hline Sexo & $\mathrm{F}$ & $\mathrm{F}$ & $\mathrm{F}$ & $\mathrm{F}$ & $M$ \\
\hline Raça & parda & branca & parda & parda & parda \\
\hline Doenças sistêmicas & HAS e DM & HAS & - & - & HAS \\
\hline Corticoterapia sistêmica* & Sim & Não & Sim & Não & Sim \\
\hline Olho injetado & OD & $\mathrm{AO}$ & $\mathrm{AO}$ & $\mathrm{AO}$ & $\mathrm{AO}$ \\
\hline Variação da PIO (mmHg) & $12-18$ & $14-17$ & $16-20$ & $12-14$ & $12-19$ \\
\hline Tempo de seguimento (meses) & 12 & 10 & 5 & 6 & 6 \\
\hline \multicolumn{6}{|c|}{$\begin{array}{l}\mathrm{F}=\text { feminino; } M=\text { masculino; } \mathrm{DM}=\text { Diabetes mellitus; } \mathrm{HAS}=\text { Hipertensão arterial sistêmica; } \mathrm{OD}=\text { olho direito; } \mathrm{AO}=\text { ambos os olhos. } \\
\text { " Uso de corticoterapia sistêmica ao mesmo tempo, sendo iniciada a progressiva diminuição da dose da medicação sistêmica logo após a injeção. } \\
\text { \# Caso de descolamento seroso de retina recidivado pela terceira vez }\end{array}$} \\
\hline
\end{tabular}




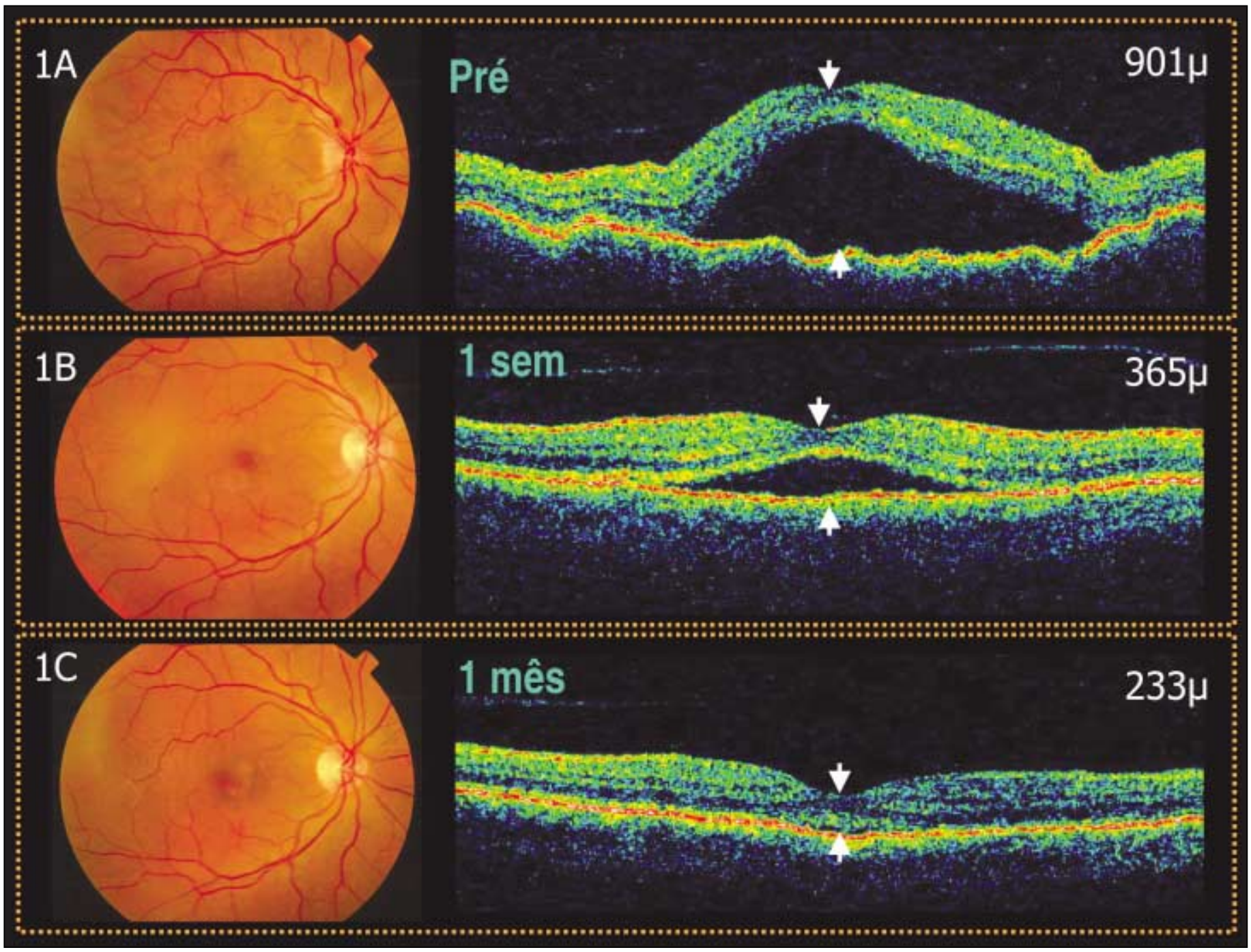

Figura 1 - Paciente 3. (Olho Direito): Retinografia e o "scan" horizontal de $6,00 \mathrm{~mm}$ da tomografia de coerência óptica centrado na região foveal. 1A- (Pré-injeção) A retinografia revelou uma hiperemia papilar associada a múltiplas áreas de descolamento seroso de retina. A tomografia de coerência óptica demonstrou o descolamento de retina com acentuada elevação retiniana associada a espessamento intra-retiniano. A altura do descolamento medida no centro da região foveal foi de $901 \mathrm{~m}$. 1B- Uma semana após a injeção do acetato de triancinolona, nota-se a persistência do fluido intra-retiniano associada a uma diminuição acentuada do descolamento seroso de retina. A altura do descolamento seroso de retina medida no centro da região foveal foi de $365 \mathrm{~m}$. 1C- Após um mês da injeção, observa-se a completa resolução do quadro exsudativo, com recuperação da anatomia retiniana normal. A altura da retina medida no centro da região foveal foi de $233 \mathrm{~m}$

retiniano difuso bilateral refratários a $80 \mathrm{mg}$ de prednisona sistêmica há 3 semanas. Sendo ambos os olhos tratados com a injeção intravítrea do acetato de triancinolona, com diminuição progressiva da dose da medicação sistêmica, até a sua retirada total em torno de 2 meses após a injeção, evoluindo, então, com a recuperação do aspecto retiniano anatômico e funcional. Está no sexto mês de seguimento sem recidivas.

Em todos os olhos a tomografia de coerência óptica revelou uma diminuição marcada no descolamento seroso de retina na primeira semana após a injeção intravítrea do acetato de triancinolona, apresentando uma altura média do descolamento seroso de retina de $770 \mu$ antes da injeção, de $311,5 \mu$ após uma semana e de $192,2 \mu$ após um mês do procedimento, evoluindo com subseqüente recuperação da acuidade visual e das características anatômicas retinianas normais (Tabela 2). Não foram observadas complicações durante o seguimento, que variou de 5 a 12 meses (média de 7,8 meses).

\section{COMENTÁRIOS}

A síndrome de Vogt-Koyanagi-Harada, assim como, outras uveítes graves, geralmente necessitam do uso da corticoterapia sistêmica, apresentam dificuldade de controle do processo inflamatório pela cronicidade do quadro e necessidade de terapia prolongada. A administração de corticóide sistêmico requer que níveis plasmáticos extremamente altos sejam alcançados $(1 \mathrm{mg} / \mathrm{kg} / \mathrm{d} 2-3$ semanas) para que níveis terapêuticos intra- 


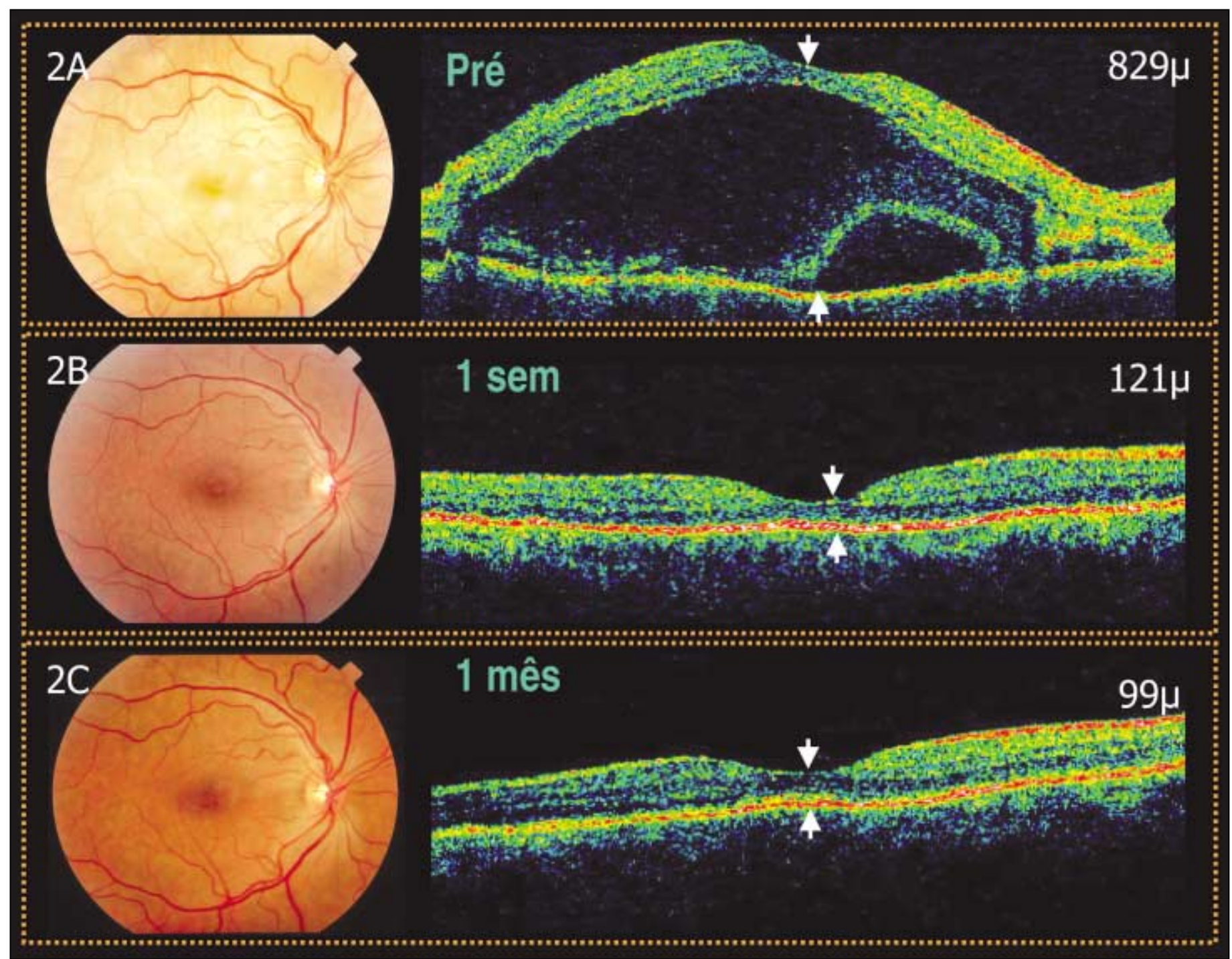

Figura 2 - Paciente 4. (Olho Direito): Retinografia e o "scan" horizontal de 6,00 mm da tomografia de coerência óptica centrado na região foveal. $2 A-$ (Pré-injeção) A retinografia revelou múltiplas áreas de descolamento seroso de retina. A tomografia de coerência óptica demonstrou o descolamento seroso de retina associado ao descolamento do epitélio pigmentado da retina e a espessamento intra-retiniano. A altura do descolamento seroso de retina medida no centro da região foveal foi de $829 \mathrm{~m}$. 2B- Uma semana após a injeção do acetato de triancinolona, a resolução completa do descolamento seroso de retina foi observada. A altura da retina medida no centro da região foveal foi de $121 \mathrm{~m}$. $2 \mathrm{C}$ - Após um mês da injeção, observase a recuperação total da anatomia retiniana normal. A altura da retina medida no centro da região foveal foi de $99 \mathrm{~m}$

oculares sejam obtidos ${ }^{(2)}$. Esses altos níveis plasmáticos, freqüentemente, levam a efeitos sistêmicos adversos graves, fazendo com que a corticoterapia sistêmica tenha de ser monitorada periodicamente ${ }^{(6,10-11)}$. A forma mais eficiente de liberação de uma droga no segmento posterior se dá por meio de sua administração diretamente no corpo vítreo ${ }^{(12)}$.

$\mathrm{O}$ acetato de triancinolona é um potente esteróide que vem sendo usado há décadas para o tratamento de inflamações oculares através de injeções peribulbares e subtenonianas ${ }^{(6)}$. A toxicidade e farmacocinética da injeção intravítrea do acetato de triancinolona foi previamente analisada em estudos com animais, onde se mostrou segura ${ }^{(12-13)}$. As complicações intravítreas mais comuns podem ser atribuídas ao próprio procedimento da injeção ou aos efeitos colaterais dos esteróides, como catarata e glauco- $\mathrm{ma}^{(7-8,12-13)}$. É uma solução hidrofóbica relativamente insolúvel que parece manter níveis terapêuticos no vítreo por mais de três meses. A injeção intravítrea de triancinolona vem sendo usada para tratar diversas entidades, como membrana neovascular subretiniana $^{(14-15)}$, edema macular diabético ${ }^{(16)}$, e para o tratamento de uveítes, revelando bons resultados visuais ${ }^{(7-8,17)}$.

Imagens seqüenciais obtidas pela tomografia de coerência óptica foram usadas para demonstrar alterações anatômicas envolvidas na progressão do descolamento seroso da retina associadas à síndrome de Vogt-Koyanagi-Harada, tais como: espessamento retiniano devido ao acúmulo de fluido intraretiniano; características do fluido subretiniano, que apresentou uma densidade óptica aumentada, quando comparada a outras causas de descolamento seroso da retina, devido a 


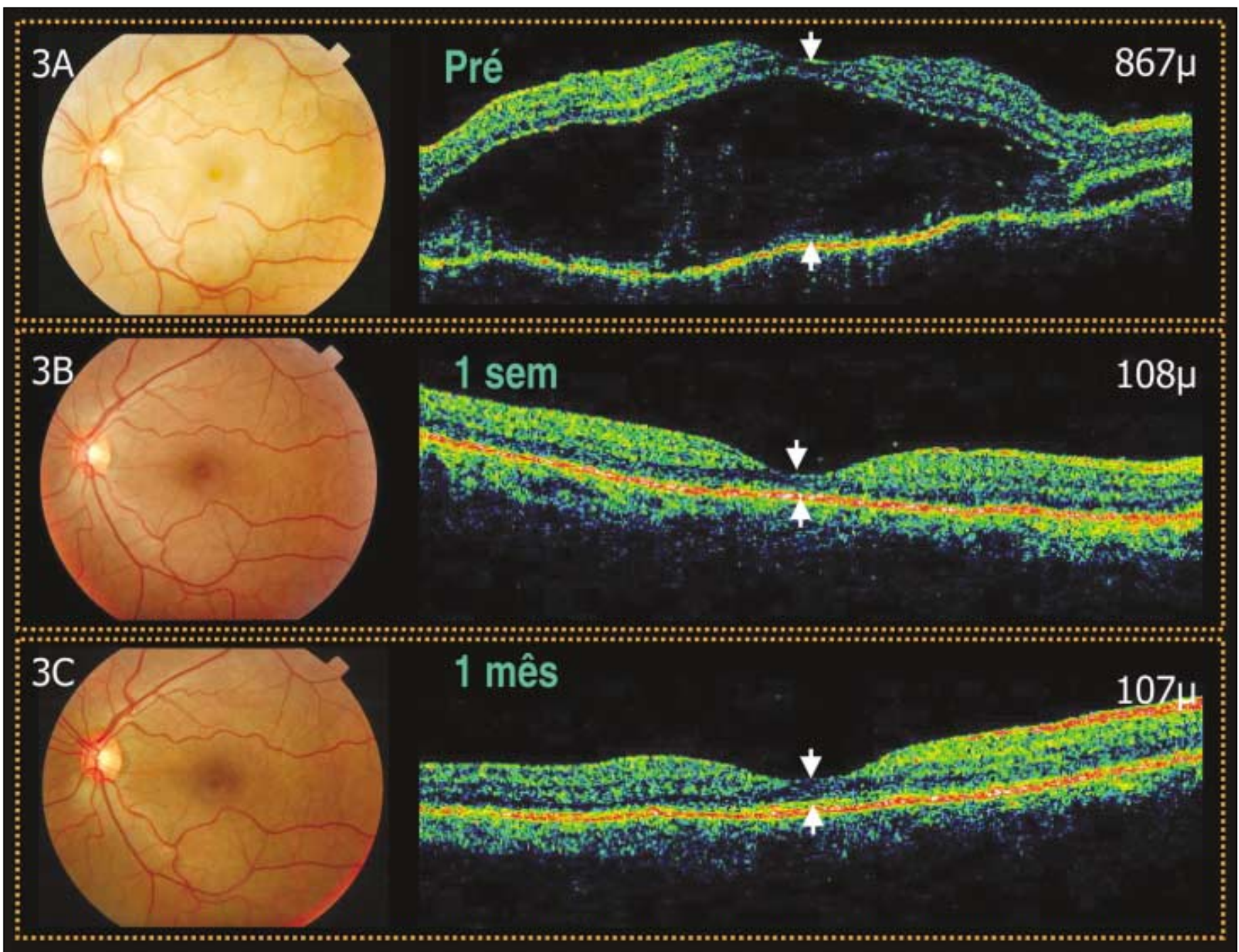

Figura 3 - Paciente 4. (Olho Esquerdo): Retinografia e o "scan" horizontal de 6,00 mm da tomografia de coerência óptica centrado na região foveal. 3A- (Pré-injeção) A retinografia revelou múltiplas áreas de descolamento seroso de retina. A tomografia de coerência óptica demonstrou o descolamento seroso de retina associado a espessamento intra-retiniano. A altura do descolamento seroso de retina medida no centro da região foveal foi de $867 \mathrm{~m}$. 3B- Uma semana após a injeção do acetato de triancinolona, a resolução completa do descolamento seroso de retina foi observada. A altura da retina medida no centro da região foveal foi de $108 \mathrm{~m}$. 3C- Após um mês da injeção, observa-se a recuperação total da anatomia retiniana normal. A altura da retina medida no centro da região foveal foi de $107 \mathrm{~m}$

provável concentração aumentada de células inflamatórias; presença de múltiplos descolamentos do epitélio pigmentado da retina em alguns casos, além de acompanhar a resolução do descolamento com medidas numéricas confiáveis da altura da retina, proporcionando uma avaliação quantitativa e objetiva da resposta ao tratamento.

\section{CONCLUSÕES}

Todos os casos aqui apresentados foram tratados com êxito com uma única injeção intravítrea de $4 \mathrm{mg}$ de acetato de triancinolona, revelando diminuição acentuada, e até, resolução total do descolamento de retina e da papilite já na primeira semana após o tratamento, mesmo nos casos sem corticotera- pia sistêmica associada ${ }^{(18)}$. Nenhuma complicação foi observada nesta série de casos.

Após o primeiro mês do tratamento os pacientes já apresentavam a recuperação das características estruturais anatômicas normais, sem sinais inflamatórios evidentes, proporcionando a diminuição progressiva da dose da corticoterapia sistêmica, o menor uso e por menos tempo, sendo que em alguns pacientes sem sintomatologia sistêmica, a medicação oral não foi necessária, apresentando os mesmos resultados.

Portanto, esta série de casos demonstrou que o uso do acetato de triancinolona intravítreo pode proporcionar em curto tempo a resolução do processo inflamatório e exsudativo da retina na síndrome de Vogt-Koyanagi-Harada cursando com melhora da acuidade visual e estabilidade do quadro no perío- 


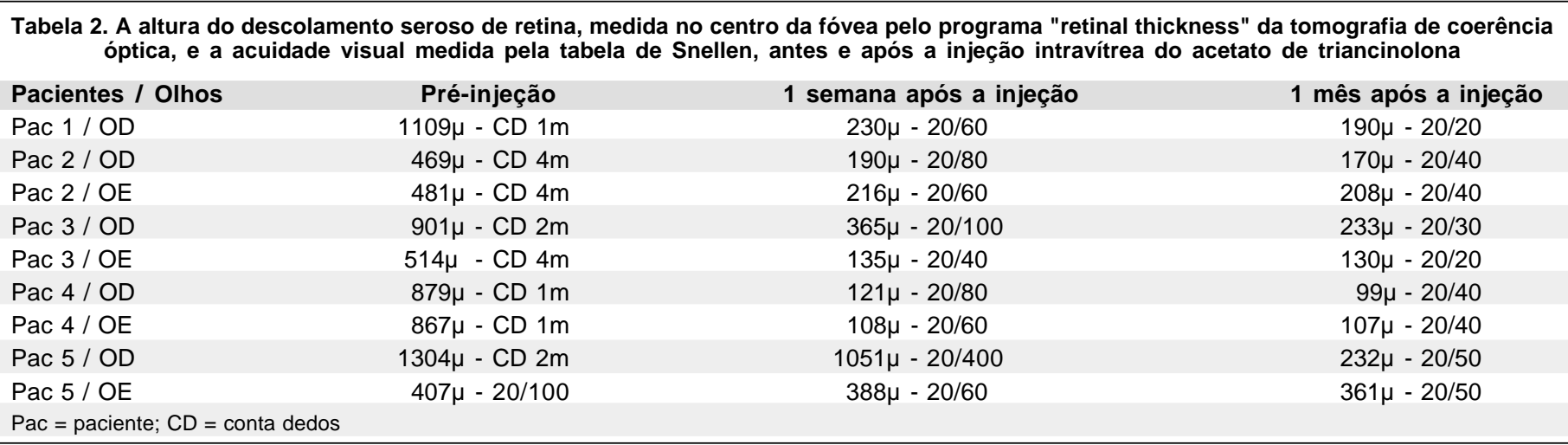

do estudado. Todavia, novos estudos são necessários avaliando a eficácia e a segurança a longo prazo deste tipo de procedimento, assim como a necessidade de retratamento.

\section{ABSTRACT}

Purpose: To report the use of intravitreal injection of triamcinolone acetonide in the acute phase of Vogt-Koyanagi-Harada's disease. Methods: Nine eyes from five patients in the acute phase of Vogt-Koyanagi-Harada's disease with serous retinal detachments were treated with a single 4-mg intravitreal injection of triamcinolone acetonide. The following parameters were evaluated: visual acuity, intraocular pressure, as well as the height of the serous retinal detachment using optical coherence tomography. Results: Optical coherence tomography images showed a marked decrease in the retinal detachment in the first week after the injection with subsequent return to normal retinal thickness in all eyes. Follow-up ranged from 5 to 12 months with a mean of 7.8 months. No complications were observed. Conclusions: Intravitreal triamcinolone acetonide provides short-term improvement in visual acuity and serous retinal detachments associated with VogtKoyanagi-Harada's disease. These findings should be followed by further studies to evaluate long-term effects.

Keywords: Retinal detachment; Uveomeningoencephalitic syndrome/drug therapy; Triamcinolone/therapeutic use; Tomography, optical coherence

\section{REFER̂̂NCIAS}

1. Bloch-Michel E, Nussenblatt RB. International Uveitis Study Group recommendations for the evaluation of intraocular inflammatory disease. Am J Ophthalmol 1987;103:234-5.
2. Oréfice F. Síndrome de Vogt-Koyanagi-Harada. In: Oréfice F, Belfort Jr. R, editors. Uveítes. São Paulo: Roca; 1987. p.295-300.

3. Belfort Junior R. Behcet, Vogt-Koyanagui-Harada e esclero-uveítes. Arq Bras Oftalmol 1981;44:87-9.

4. Belfort Junior. R, Nishi M, Hayashi S, Abreu MT, Petrilli AM, Plut RC. Vogt-Koyanagi-Harada's disease in Brazil. Jpn J Ophthalmol 1988;32:344-7.

5. Beniz J, Forster DJ, Lean JS, Smith RE, Rao NA. Variations in clinical features of the Vogt-Koyanagi-Harada syndrome. Retina 1991;11:275-80.

6. Rubsamen PE, Gass JD. Vogt-Koyanagi-Harada syndrome. Clinical course, therapy and long-term visual outcome. Arch Ophthalmol 1991;109:682-7.

7. Degenring RF, Jonas JB. Intravitreal injection of triamcinolone acetonide as treatment for chronic uveitis. Br J Ophthalmol 2003;87:361.

8. Antcliff RJ, Spalton DJ, Stanford MR, Graham EM, Fytche TJ, Marshall J. Intravitreal triamcinolone for uveitic cystoid macular edema: an optical coherence tomography study. Ophthalmology 2001;108:765-72.

9. Yamanaka E, Ohguro N, Yamamoto S, Nakagawa Y, Imoto Y, Tano Y. Evaluation of pulse corticosteroid therapy for Vogt-Koyanagi-Harada disease assessed by optical coherence tomography. Am J Ophthalmol 2002; 134:454-6.

10. Skalka HW, Prchal JT. Effect of corticosteroids on cataract formation. Arch Ophthalmol 1980;98:1773-7.

11. Schwartz B. The response of ocular pressure to corticosteroids. Ophthalmol Clin North Am 1996;6:929-89.

12. McCuen BW 2nd, Bessler M, Tano Y, Chandler D, Machemer R. The lack of toxicity of intravitreally administered triamcinolone acetonide. Am J Ophthalmol 1981;91:785-8.

13. Schindler RH, Chandler D, Thresher R, Machemer R. The clearance of intravitreal triamcinolone acetonide. Am J Ophthalmol 1982;93:415-7.

14. Challa JK, Gillies MC, Penfold PL, Gyrory JH, Hunyor AB, Billson FA. Exudative macular degeneration and intravitreal triamcinolone: 18 month follow up. Aust N Z J Ophthalmol 1998;26:277-81.

15. Penfold PL, Gyory JF, Hunyor AB, Billson FA. Exudative macular degeneration and intravitreal triamcinolone. A pilot study. Aust N Z J Ophthalmol 1995;23:293-8.

16. Martidis A, Duker JS, Greenberg PB, Rogers AH, Puliafito CA, Reichel E, Baumal C. Intravitreal triamcinolone for refractory diabetic macular edema. Ophthalmology 2002;109:920-7.

17. Martidis A, Duker JS, Puliafito CA. Intravitreal triamcinolone for refractory cystoid macular edema secondary to birdshot retinochoroidopathy. Arch Ophthalmol 2001;119:1380-3.

18. Andrade RE, Muccioli C, Farah ME, Nussenblatt RB, Belfort R Jr. Intravitreal triamcinolone injection in the treatment of serous retinal detachment in VogtKoyanagi-Harada syndrome. Am J Ophthalmol 2004;137:572-4. 